\title{
Prevalência da cisticercose bovina no estado do Paraná, sul do Brasil: avaliação de 26.465 bovinos inspecionados no SIF 1710
}

\section{Bovine cysticercosis prevalence in Parana state, southern of Brazil, in animals slaughtered under the SIF 1710}

\author{
Valmir Kowaleski Souza ${ }^{1}$; Maria do Carmo Pessôa-Silva ${ }^{2}$; \\ João Carlos Minozzo ${ }^{3}$; Vanete Thomaz-Soccol ${ }^{3 *}$
}

\section{Resumo}

\begin{abstract}
A cisticercose é a patologia mais encontrada na Inspeção post mortem em bovinos abatidos em estabelecimentos com Serviço de Inspeção Federal (SIF). No Brasil a média da prevalência desta parasitose é de 5\%, porém, estes índices podem variar segundo a região anatômica avaliada, região geográfica ou período de realização de trabalho. Para conhecer a prevalência desta patologia em bovinos, no Estado do Paraná, foi realizada uma pesquisa durante seis meses, entre julho a dezembro de 2000, em animais procedentes de 137 municípios, que foram abatidos no Frigorífico Argus, Região Metropolitana de Curitiba. A prevalência de animais positivos encontrados com Cysticercus bovis variou de 0 a 27,27\% com média de 3,83\% entre os 26.465 bovinos inspecionados. Os municípios com maior número de animais abatidos tiveram prevalência aparente no lote entre 2,8 a $5 \%$. De acordo com o sexo dos animais não foi verificada predileção ou especificidade parasitária sendo que nos machos foi encontrado o índice de $3,81 \%$ e nas fêmeas 3,93\%. Todos os cisticercos encontrados vivos passaram pelo processo de desinvaginação, coloração por carmim acético e identificação microscópica constatando que 100\% eram C. bovis. As implicações epidemiológicas destes achados são discutidas no presente trabalho. Palavras-chave: Cysticercus bovis, cisticercose bovina, zoonoses, prevalência, epidemiologia
\end{abstract}

\begin{abstract}
Cystercosis is the most frequently detected pathology in post mortem inspection in bovine slaughtered at establishments under the Federal Inspection Service (SIF). In Brazil, the average prevalence of this parasitosis is 5\%, but the rates can vary according to the anatomic area screened, the geographic region, or the period of time the work was carried out. In order to find out the prevalence of this pathology among bovine, in the State of Parana-Brazil, a six months research was carried out - from July to December, 2000 - in animals originating from 137 towns and slaughtered in the Argus Cold Store, located in the Metropolitan Area of Curitiba. The prevalence of positive animals found with Cysticercus bovis varied from 0 to $27.27 \%$ with an average of $3.83 \%$ among the $26465 \mathrm{animals}$ inspected. The towns with larger numbers of slaughtered animals had an apparent prevalence in allotment between $2.8 \%$ to $5 \%$. Regarding sex, a $3.81 \%$ rate was found among males and $3.93 \%$ among females, but no parasitary specificity or preference were detected. All cystercercus found alive were submitted to the desinvagination process, staining with acetic carmin, and microscopic identification evidencing that $100 \%$ were C. bovis. The epidemiological implications of these findings are discussed in the present work.

Key words: Cysticercus bovis, bovine cystercosis, zoonoses, prevalence, epidemiology
\end{abstract}

\footnotetext{
1 Superintendente Federal do Ministério da Agricultura no Estado do Paraná e Mestre em Ciências Veterinárias, UFPR.

2 Área de Epidemiologia da Secretaria de Estado da Agricultura do Paraná.

3 Professor Associado de Parasitologia Veterinária -Universidade Federal do Paraná, Departamento de Patologia Básica, Centro Politécnico.

* Autor para correspondência
} 


\section{Introdução}

A cisticercose bovina é causada pelo metacestoda Cysticercus bovis que está presente na musculatura do bovino, hospedeiro intermediário desta parasitose. O homem, hospedeiro definitivo, alberga o parasito adulto no intestino delgado e elimina ovos ou proglotes do parasito, contaminando o ambiente. As ações preventivas de controle do complexo teníase/ cisticercose baseiam-se num conjunto de medidas que visam impedir a infecção do homem pela Taenia saginata e, com isto, bloquear o ciclo desse parasito na natureza.
Atualmente o recurso de maior expressão é a inspeção de carnes com exame post mortem criterioso, o julgamento e o saneamento adequado das carcaças parasitadas. Assim, a inspeção de carne é a medida direta de maior importância na prevenção da teníase, pois apesar de suas limitações a inspeção identifica bem as carcaças com infecções intensas e leves, e serve também como advertência precoce de infecção em uma comunidade.

No Brasil os dados de prevalência desta zoonose apresentam variação entre os estados e período de avaliação. As tabelas 1 e 2 resumem os dados de prevalência encontrados em registros bibliográficos.

Tabela 1. Prevalência da Cisticercose bovina em animais abatidos por estado, segundo autor e período analisado.

\begin{tabular}{llccc}
\hline Estado & Autor /Ano & Período analisado & $\begin{array}{c}\text { Prevalência de } \\
\text { cisticercose bovina }\end{array}$ & $\begin{array}{c}\text { Fonte da } \\
\text { informação }\end{array}$ \\
\hline Rio Grande do Sul & Rodrigues (1993) & 1980 a 1990 & $4,51 \%$ & SIPA/DFA-RS \\
Minas Gerais & Reis (1996) & 1979 a 1993 & $2,79 \%$ & \\
Goiás & Reis (1996) & 1979 a 1993 & $1,05 \%$ & \\
Mato Grosso & Reis (1996) & 1979 a 1993 & $0,44 \%$ & \\
Minas Gerais & Souza (1997) & 1990 a 1994 & $4,15 \%$ & SIF - DIPOA \\
\hline
\end{tabular}

Tabela 2. Prevalência da Cisticercose bovina em animais inspecionados pelo DIPOA, por estado, Brasil.

\begin{tabular}{lcc}
\hline \multicolumn{1}{c}{ Estado } & Número de animais abatidos & $\begin{array}{c}\text { Prevalência } \\
(\mathbf{\%})\end{array}$ \\
\hline São Paulo & 197.407 & 2,87 \\
Minas Gerais & 12.593 & 2,18 \\
Mato Grosso do Sul & 292.548 & 1,46 \\
Mato Grosso & 4.737 & 0,42 \\
TOTAL & $\mathbf{5 0 7 . 2 8 5}$ & $\mathbf{1 , 7 3}$ \\
\hline
\end{tabular}

Fonte: Fernandes et al. (2002). 
Em levantamento estatístico dos dados nosográficos do Serviço de Inspeção Federal do Ministério da Agricultura, DFA-PR e de algumas Inspeções Federais isoladas, a prevalência de cisticercose em suínos no estado do Paraná variou de 5 a 10\% na década de 50 para 0,001 em 1999. Enquanto que em bovinos os índices eram de 2 a 2,50 nos anos 50 e passaram para 4,60 a 7,70\% em 1999 (Tabela 3).

Tabela 3. Prevalência da cisticercose animal no estado do Paraná no período de 1950 à 1999.

\begin{tabular}{ccc}
\hline Anos & $\begin{array}{c}\text { Cysticercus cellulosae } \\
\text { em suínos (\%) }\end{array}$ & $\begin{array}{c}\text { Cysticercus bovis } \\
\text { em bovinos (\%) }\end{array}$ \\
\hline $50-52$ & 5 à 10 & 2 a 2,50 \\
$66-67$ & 4,20 & 2,50 \\
$79-82$ & 0,46 & 7,18 \\
84 & 0,30 & 2,78 a 3,60 \\
$90-95$ & 0,03 & 3,60 a 5,10 \\
96 & 0,01 & 3,66 \\
97 & 0,01 & 3,68 \\
98 & 0,001 & 4,77 \\
99 & 0,001 & 4,60 a 7,70 \\
\hline
\end{tabular}

Fonte: SIPA-DFA-PR.

Pouco se conhece da cisticercose bovina nos diferentes municípios paranaenses. Assim, visando atualizar as informações sobre prevalência aparente em bovinos oriundos de Municípios do Estado do Paraná, abatidos no SIF 1710 - Argus Ltda, localizado em São José dos Pinhais/Pr, foram inspecionados 26.465 bovinos procedentes de 137 municípios do estado.

\section{Material e Métodos}

Foram inspecionados 26.465 bovinos, na maioria animais azebuados, compreendendo machos e fêmeas e com faixas etárias variando entre 18 a 60 meses. Os animais procediam de 137 municípios do Estado do Paraná, devidamente identificados e abatidos no período de julho a dezembro de 2000, no matadouro - frigorífico Argus Ltda., SIF 1710, localizado na região metropolitana de Curitiba, no município de São José dos Pinhais/ PR.

Os animais foram abatidos conforme tecnologia de produção padrão para bovinos. Os trabalhos de inspeção nas linhas foram feitos por uma equipe composta de sete agentes de Inspeção devidamente treinados para realizar a inspeção post mortem, sob supervisão e responsabilidade do Médico Veterinário do Serviço de Inspeção Federal (SIF). Os exames de rotina desenvolvidos na pesquisa da cisticercose bovina nas linhas de inspeção (cabeça, língua, coração, diafragma e esôfago), basearam-se nas normas padronizadas pelo SIF, (BRASIL, 1971, 1980, 1988, 2006).

Caso fossem encontrados cisticercos nos animais nas linhas de inspeção, as lesões eram identificadas e as meias-carcaças, juntamente com as vísceras e cabeça eram encaminhadas para o D.I.F. (Departamento de Inspeção Final) onde eram examinadas pelo Médico Veterinário, conforme procedimentos regulamentares. Os dados eram anotados em papeleta específica do D.I.F.

Ao serem encontrados no D.I.F. os cisticercos eram coletados, acondicionados em sacos plásticos devidamente identificados de acordo com a sua procedência: data do abate, $\mathrm{n}^{\circ}$ de animais do lote, quantidade total de animais abatidos no dia, classificação do cisto de acordo com seu estado de calcificação ou não, localização anatômica, $n^{\circ}$ de cistos encontrados na carcaça, idade e sexo do animal. Quando na inspeção post mortem não foi observada a presença de cisto, o animal foi considerado negativo para cisticercose e liberados. 
Os cistos encontrados foram levados ao laboratório e classificados em duas categorias: vivos e degenerados. A identificação dos cisticercos foi feita após o processo de desinvaginação dos cistos vivos, os quais foram processados individualmente. Em seguida foi feita sua fixação em lâmina, por leve compressão, e então colocada em placas de Petri, que continham líquido de Railliet e Henry e ai permaneciam por 24 horas. O líquido, por capilaridade, entrava em contato com a larva fixandoa. Os líquidos fixadores funcionam também como conservantes das larvas, até sua coloração.

O método usado para coloração foi o do Carmim Clorídrico. Em seguida, as larvas foram identificadas por meio de microscopia óptica com aumentos de 100 e 400X - Olympus (mod- CBAK), visualizandose o protoescólex, colo e pseudo-estróbilo, diferenciando-se o C. bovis de C. celullosae pela ausência de dupla coroa de ganchos.

\section{Resultados}

A avaliação de 26.465 procedentes de 137 Municípios, no período da avaliação permitiu identificar 1.014 animais positivos representando uma ocorrência de 3,83\% animais cisticercósicos. Na tabela 4 são registrados os dados de ocorrência mensal, número de animais abatidos e a prevalência de cisticercose.

Na figura 1 é dada a distribuição espacial da cisticercose bovina no Paraná, onde se observa que na maioria dos municípios a prevalência aparente nos lotes está entre 1,0 a 5,0\%.

Ao analisar os resultados, por procedência e no período trabalhado, observa-se que dos 137 municípios que tiveram bovinos abatidos no SIF 1710, somente $2,86 \%$ deles (com origem em 22 municípios) não foram identificados animais cisticercósicos, sendo que os que tiveram animais com cisticercose as prevalências aparentes encontradas nos animais abatidos variaram de $0,29 \%$ a $27,27 \%$.

Tabela 4. Prevalência da cisticercose observada mensalmente no abate de bovinos realizados no SIF 1710, julho a dezembro de 2000.

\begin{tabular}{lccc}
\hline \multicolumn{1}{c}{ Mês } & $\mathbf{N}^{\circ}$ de Animais Abatidos & $\mathbf{N}^{\circ}$ de casos Positivos & Prevalência em \% \\
\hline Julho & 5.291 & 149 & 2,82 \\
Agosto & 4.299 & 170 & 3,95 \\
Setembro & 4.578 & 194 & 4,23 \\
Outubro & 4.422 & 184 & 4,16 \\
Novembro & 4.006 & 176 & 4,39 \\
Dezembro & 3.869 & 141 & 3,65 \\
Total & $\mathbf{2 6 . 4 6 5}$ & $\mathbf{1 . 0 1 4}$ & $\mathbf{3 , 8 3}$ \\
\hline
\end{tabular}

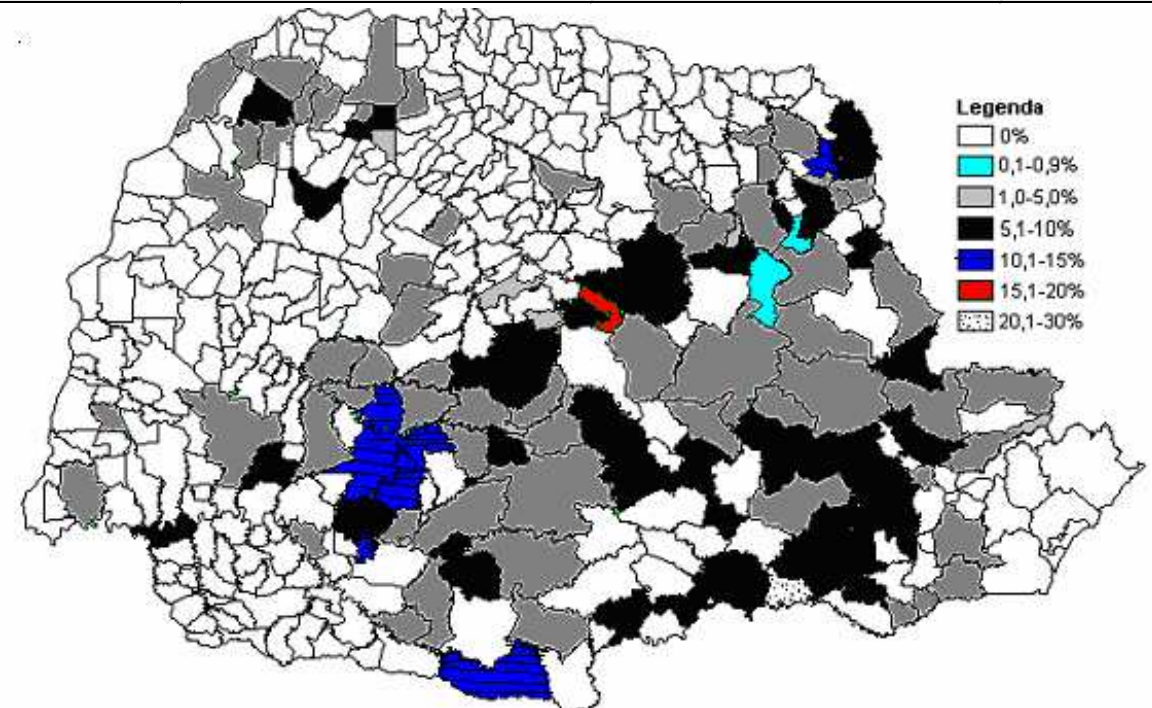

Figura 1. Distribuição espacial da ocorrência da cisticercose em bovinos do Estado do Paraná abatidos no SIF 1710 junho a dezembro de 2000. 
Os municípios que tiveram maior quantidade de animais para abate, no mesmo período, ou seja, que tiveram melhor representatividade amostral foram onze. Nestes a prevalência aparente da cisticercose nos rebanhos prontos para abate variou de 0,29 a 5,25 (Tabela 5). No entanto, quando se considera a maior prevalência aparente, 10 municípios apresentaram índices entre 9,47 a 27,27\% (Tabela 6).

Quanto ao sexo dos animais abatidos e avaliados foi encontrada uma prevalência aparente eqüitativa de cisticercos, sendo que nos machos foi encontrado o índice de 3,81\% e nas fêmeas 3,93\%, o que demonstra não haver predileção ou especificidade parasitária (Figura 2).

Já na análise da faixa etária verificou-se que animais entre 18 a 36 meses tiveram prevalência de $4,50 \%, 36$ a 48 meses a prevalência foi de $2,32 \%$ e os animais acima de 48 meses apresentaram índices de $3,63 \%$ (Tabela 7).

Tabela 5. Prevalência aparente e em relação ao total abatido de cisticercose em bovinos, nos lotes dos 10 municípios com maior número de animais abatidos no SIF 1710, julho a dezembro de 2000.

\begin{tabular}{lcc}
\hline \multicolumn{1}{c}{ Município } & Prevalência Aparente no Lote (\%) & $\begin{array}{c}\text { Representatividade do Município } \\
\text { em relação ao total abatido (\%). }\end{array}$ \\
\hline Palmeira & 4,37 & 5,45 \\
Paranavaí & 4,87 & 4,81 \\
Guarapuava & 3,25 & 4,42 \\
Candói & 4,82 & 4,31 \\
Palmital & 2,78 & 4,08 \\
Nova Laranjeiras & 0,29 & 3,91 \\
Planaltina do Paraná & 2,77 & 3,82 \\
Ponta Grossa & 5,25 & 2,30 \\
Teixeira Soares & 3,72 & 2,23 \\
Piraí do Sul & 2,72 & 2,22 \\
Honório Serpa & 3,76 & 2,11 \\
\hline
\end{tabular}

Tabela 6. Municípios paranaenses que apresentaram maiores prevalências aparente de cisticercose bovina

\begin{tabular}{lcc}
\hline \multicolumn{1}{c}{ Município } & $\begin{array}{c}\text { Prevalência Aparente no Lote } \\
(\boldsymbol{\%})\end{array}$ & $\begin{array}{c}\text { Representatividade do município, em } \\
\text { relação ao total de animais abatidos no } \\
\text { período. } \\
(\boldsymbol{\%})\end{array}$ \\
\hline Campo Largo & 9,47 & 0,36 \\
Capanema & 10,0 & 0,08 \\
Rosário do Ivaí & 18,18 & 0,08 \\
Japira & 10,0 & 0,08 \\
Joaquim Távora & 10,87 & 0,35 \\
Laranjeiras do Sul & 13,82 & 1,04 \\
Rio Bonito do Iguaçu & 9,88 & 0,31 \\
Palmas & 13,33 & 0,11 \\
Saudades do Iguaçu & 11,58 & 0,35 \\
Antônio Olinto & 27,27 & 0,04 \\
\hline
\end{tabular}


Tabela 7. Prevalência aparente de cisticercose bovina, no estado do Paraná, segundo a faixa etária

\begin{tabular}{llll}
\hline $\begin{array}{l}\text { Idade } \\
\text { em meses }\end{array}$ & $\begin{array}{l}\mathbf{N}^{\mathbf{0}} \text { animais abatidos } \\
\text { na faixa etária }\end{array}$ & $\begin{array}{l}\mathbf{N}^{\mathbf{0}} \text { animais } \\
\text { cisticercósicos }\end{array}$ & $\begin{array}{c}\text { Prevalência } \\
\text { Aparente }\end{array}$ \\
\hline 18 a $36 \mathrm{~m}$ & 15.729 & 715 & $4,54 \%$ \\
36 a $48 \mathrm{~m}$ & 7.569 & 184 & $2,42 \%$ \\
$>48 \mathrm{~m}$ & 3.168 & 115 & $3,64 \%$ \\
\hline Total & 26.465 & 1.014 & $3,83 \%$ \\
\hline
\end{tabular}

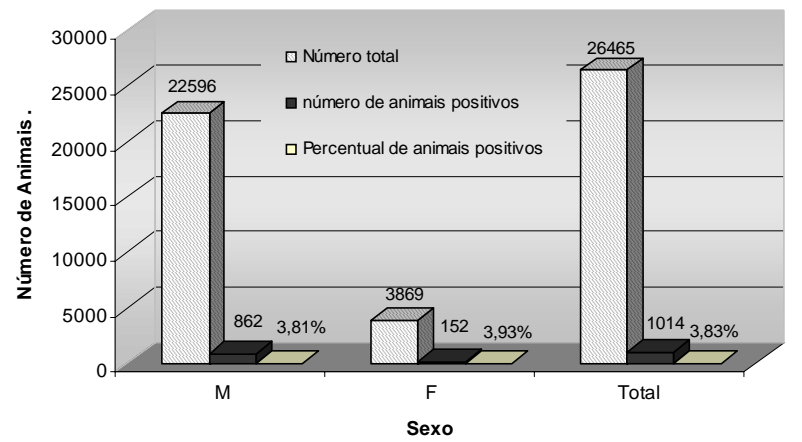

Figura 2. Número de bovinos abatidos, número e percentual de portadores de cisticercos, por sexo.

O total de cistos vivos encontrados foi 359, o que representou 33,03\%. Todos foram submetidos à técnica de desinvaginação, coloração por Carmim Clorídrico, fixados entre lâmina e lamínula e submetidos à identificação morfológica em microscópio óptico nos aumentos de 100 e 400 vezes. Todos os cisticercos viáveis possuíam escólex quadrangular e eram desprovidos de dupla coroa de acúleos sendo identificados como C. bovis (Figura 3).

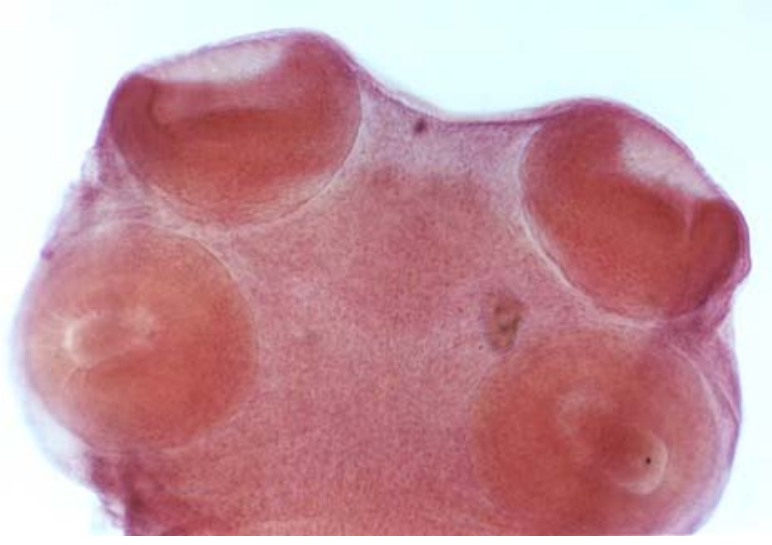

Figura 3. Fotomicrografia (400X) de Escolex do metacestoda de Taenia saginata observados em bovinos após inspeção.

\section{Discussão}

No presente estudoforam inspecionados 26.465 animais oriundos de 137 municípios do PR, correspondendo a aproximadamente a $3^{\mathrm{a}}$ parte da área geopolítica do estado, que é composto por 399 municípios. A prevalência aparente encontrada nos lotes abatidos foi de 3,83\%, com dados variando de município para município, ou mesmo de propriedade para propriedade. A análise dos resultados, por procedência dos animais abatidos, no período pesquisado, constatou-se que dos 137 municípios que enviaram lotes para abate no período analisado pelo SIF 1710, 10 apresentaram as maiores prevalências de cisticercose bovina superando 9\%. Considerando que a amostra coletada ficou limitada à área de abrangência do Frigorífico Argus e a população alvo era apenas aquela pronta para abate, a amostragem pode ter sido dirigida ao invés de ter uma distribuição aleatória, o que pode ter se tornado um viés de seleção da amostra. Apesar disso, a grande distribuição espacial da enfermidade, sugere uma influência de fatores com grande poder de difusibilidade como, por exemplo, água contaminada de rios, que devem receber ao longo de seu percurso esgotos urbanos. Desta forma, contaminam as pastagens por ocasião dos constantes alagamentos ou pela ingestão direta da água contaminada pelos ovos de $T$. saginata. Tal hipótese pode ser embasada também, pela diversidade de sistemas de produção da pecuária, encontrado nos municípios que tiveram animais com maiores índices de infecção pela cisticercose e com representatividade dos lotes do município entre 2\% e 5,45\%. Municípios com predominância de criação intensiva (confinamentos 
e semi-confinamentos) tiveram alta prevalência como Paranavaí (4,81\%), Palmeira (4,87\%) e Guarapuava (3,25\%), e de municípios com exploração predominantemente extensiva (bovinos criados livremente na pastagem), podemos citar os municípios de Capanema e Japira (10,00\%), Joaquim Távora (10,87\%), Laranjeiras do Sul (13,82\%) e Rosário do Ivaí (18,18\%).

Os resultados dos diferentes trabalhos publicados nas últimas décadas, (COSTA et al., 1977; DFASIPA-RS, 1980; KALIL, 1984; UNGAR et al., 1990, 1992; RODRIGUES, 1993; SANTOS, 1993a, 1993b; REIS et al., 1996; SOUZA; ANTUNES; GUSATIMOSIN, 1997; CARMO et al., 1997; CORREA et al., 1997; BIONDI; HENRIQUE; OLIVEIRA, 1999; FERNANDES et al., 2002; DFASIPA-SP, 2000; MIRANDA, 2002; PAIVA, 2005; PEREIRA; SCHWANZ; BARBOSA, 2006) mostram a presença crescente da cisticercose bovina, em todos os estados do circuito pecuário CentroOeste, Sudeste e Sul, onde se fazem necessária urgentes medidas epidemiológicas, pois demonstra infecções alarmantes em bovinos e, por conseqüência, indica a presença de $T$. saginata parasitando o homem, os quais necessitam de cuidados primários de saúde pública. A maior taxa de prevalência da cisticercose bovina em São Paulo e Minas Gerais coincidem com uma maior concentração de criações de bovinos próximos aos grandes centros urbanos e com uso intensivo de mão-de-obra temporária proveniente do meio rural.

Quanto ao sexo dos animais parasitados observouse que ambos os sexos estão expostos ao mesmo risco, uma vez que a diferença não foi estatisticamente significante. Dorny (2000) em um estudo soroepidemiológico detectou que as fêmeas leiteiras com idade superior a 5 anos apresentaram uma prevalência de 5,1\% de cisticercose e com idade entre 3 a 4 anos, 6,3\%. Comparando-se com as vacas tipo corte da mesma faixa etária, encontrou respectivamente $4 \%$ e $3,9 \%$; induzindo a interpretação de que o manejo e o contato mais permanente do homem com as vacas leiteiras do que com as de corte, resulta numa maior contaminação ambiental e em conseqüência uma alta prevalência da cisticercose. No mesmo estudo Dorny (2000) encontrou uma prevalência de 1,2\% de cisticercose para os machos de bovinos destinados ao corte na faixa etária compreendida entre 1 a 2 anos e de 5\% para 3 a 4 anos. O desenvolvimento da imunidade é possível que afete a relação entre idade e prevalência. Em áreas onde a transmissão da T. saginata entre o homem e o bovino é freqüente, é mais provável que o bovino se infecte ainda jovem e desenvolva imunidade contra reinfecções. Contrastando, com áreas onde a transmissão é mais esporádica, o tempo de exposição pode ser mais importante.

Quanto à identificação dos cisticercos provenientes de bovinos naturalmente infectados não foram detectadas alterações morfológicas que pudessem identificá-los como sendo originados de ovos de T. solium. Segundo Borchert, 1981; Lapage, 1981 e Pessoa e Martins, 1982 vários animais podem ser hospedeiros intermediários da T. solium, entre eles os bovinos. Gusso et al. (2000) procederam a uma infecção experimental em bovinos com ovos de T. solium e, os cisticercos resultantes desta infecção sofreram modificações em sua estrutura morfológica, apresentando acúleos rudimentares e sem a presença da dupla coroa. Pessoa (1982) descreveu que em processos de degeneração os ganchos podem ser encontrados no líquido vesicular e também cistos sem rostelo e sem coroa de ganchos. No entanto, no exame microscópico de 395 cisticercos vivos todos foram identificados como metacestoda de $T$. saginata.

A atividade da bovinocultura de corte no Brasil movimenta altas cifras econômicas já que, possuímos um rebanho de aproximadamente 180 milhões de cabeças de bovinos. Considerando-se a prevalência média brasileira da cisticercose em 5\% (SANTOS, 1993a), teríamos 9 milhões de animais infectados por este metacestoda e que estariam inviabilizados para a exportação, pois o RIISPOA e os Regulamentos técnicos da UE e EUA impedem a exportação de carne procedentes de animais portadores de doenças, 
assim como aqueles destinados ao DIF (Departamento de Inspeção Final) para uma rigorosa Inspeção Final. Os prejuízos econômicos são distribuídos ao longo da cadeia produtiva da carne. Porém, o segmento intermediário dos matadouros frigoríficos é quem detém a maior parcela, devido às regras impostas pelo mercado, principalmente dos produtores de bovinos que tradicionalmente não admitem descontos pecuniários pela infecção de seus animais (carcaças) pela cisticercose, a não ser em casos de condenações totais das carcaças (cisticercose generalizada) acompanhadas de declarações técnicas dos destinatários do Sistema Oficial de Inspeção.

Os prejuízos dos produtores rurais seriam principalmente devido à condenação total das carcaças diagnosticadas pela Inspeção; ao deságio no preço do boi, quando a venda é baseada no preço morto e estes animais seriam destinados à exportação, os frigoríficos recusam-se comprar novos lotes de animais dessas propriedades altamente infectadas.

Aos matadouros frigoríficos caberiam os prejuízos: causados pelo seqüestro de carcaças positivas para cisticercose e seu posterior destino condicional ao tratamento térmico por calor, frio e a salga; ao aumento dos custos com mão de obra dos manipuladores e eletricidade. O retorno econômico da carcaça é adiado pelas técnicas condicionais; pela condenação total das vísceras; desvalorização da carcaça recortada na pesquisa dos cisticercos. Haveria ainda, o grande risco do consumidor detectar cistos viáveis em cortes de carne resfriadas comercializadas em embalagens à vácuo com a identificação da marca comercial da carne (frigorífico) e o mesmo vir a sofrer demandas judiciais de indenizações e denúncias públicas por intermédio dos órgãos de defesa dos consumidores e ONGs.

Segundo Schantz et al. (1992), a perda econômica anual na América Latina devido à cisticercose é de aproximadamente US\$ 164 milhões. Na África as perdas por cisticercose bovina estão estimadas em US\$ 1.000 e 2.000(milhões), onde a impossibilidade prática de manter o gado livre da infecção continua frustrando o crescimento de uma indústria de carne rentável em muitos países em desenvolvimento. Estes índices podem ser ainda maiores uma vez que as prevalências da cisticercose são calculadas tendo como base o método de corte e olha que apresenta baixa sensibilidade (MINOZZO et al., 2002).

O complexo teníase/cisticercose permanece com elevados índices no Brasil principalmente no que se refere a Taenia saginata/Cysticercus bovis e vem aumentando significativamente. As infecções em humanos não são consideradas como graves, pois geralmente são bem tolerados pelo hospedeiro definitivo. No entanto, além do caráter zoonótico dessa patologia, ela também representa graves perdas econômicas para o país devido à condenação e ao aumento do custo de processamento das carcaças submetidas a aproveitamento condicional. A perda por cisticercose bovina pode ser de US\$ 25 por animal infectado. Diante do exposto é necessário tomar medidas para reduzir a prevalência da cisticercose bovina, teníase humana e da contaminação ambiental por ovos de T. saginata. É indispensável que Ministério da Agricultura, da Saúde e do Meio Ambiente tenham programas conjuntos para reduzir erradicar o complexo teníase/cisticercose.

\section{Agradecimentos}

$$
\text { CNPq e Capes - pelo apoio financeiro }
$$

\section{Referências}

BIONDI, G. F.; HENRIQUE, C. H.; OLIVEIRA, A. C. Alto índice de cisticercose bovina, em sistema de confinamento, detectado no município de Goiânia (GO): estratégia de controle através da utilização do sulfóxido de albendazol 17\%. A Hora Veterinária, Porto Alegre, v.18, n.107, p. inicial e final, 1999.

BORCHERT, A. Parasitologia veterinária. 3.ed. Zaragoza: España, Acribia, 1981.

BRASIL Ministério da Agricultura Pecuária e AbastecimentoMAPA-DIPOA. Padronização de técnicas, instalações e equipamentos. Brasília, 1971. v.1 Bovinos, p.78-95. 
BRASIL Ministério da Agricultura. Divisão de Inspeção de Carnes. Serviço de inspeção de produtos de origem animal. Brasília, 1988. Circular n.054 19 maio 1988. Trabalhos científicos sobre cisticercose.

BRASIL. Ministério da Agricultura. Regulamento de inspeção industrial e sanitária de produtos de origem animal. Brasília, 1980.

BRASIL. Ministério da Agricultura. Serviço de Inspeção Federal - SIF. Relação de abate de doença por procedência e município. Disponível em: < http://extranet. agricultura.gov.br>. Acesso em: 15 set. 2006.

CARMO, R. G.; OLIVEIRA, J. V.; BANDINI, O. R.; CARVALHO, J. O.; LIMA, R. W.; REIS, S. Prevalência de cisticercose bovina no estado de Mato Grosso do Sul. Higiene Alimentar, São Paulo, v.11, n.50, p.45-50, 1997.

CORRÊA, G. L. B.; ADAMS, A. N.; ANGNES, F. A.; GRIGOLETTO, D. S. Prevalência de cisticercose em bovinos abatidos em Santo Antônio das Missões, Rs, Brasil. Revista da Faculdade de Zootecnia, Veterinária e Agronomia,Uruguaiana, n.4, p.43-45,1997.

COSTA, A. J.; MORI-SOBRINHO, A.; AVILA, F. A.; CAVALCANTI SOBRINHO, E. Distribuição geográfica da freqüência de cisticercose bovina em algumas regiões do Estado de São Paulo - Brasil. Científica, Jaboticabal, v.5, n.3, p.370-379, 1977.

DORNY, P.; VERCAMMEN, F.; BRANDT, J.; VANTEEENKISTE, W.; BERKVENS, D.; GREETS, S. Seroepidemiological study of Taenia saginata cysticercosis in Belgian cattle. Veterinary Parasitology, Amsterdam,. v.88, n.1,p.43-49, 2000.

FERNANDES, J. O. M.; SILVA, C. L. S. P.; BORGES, J. H. R.; PEGAIANE, J. C.; COELHO, R. V. Prevalência da cisticercose bovina em animais abatidos em estabelecimento sob regime de inspeção federal no munícipio de andradina-SP. Revista Ciências Agrárias e da Saúde: FEA, Andradina, v.2, n.1, p.14-17, 2002.

GUSSO, R. L. F.; MINOZZO, J. C.; THOMAZ-SOCCOL, V.; CAMARGO, N. J.; LOPES, C. M. Experimental infection of cattle with eggs of Taenia solium. Archives of Veterinary Science, Curitiba, v.5, p.23-27, 2000.

KALIL, R. M. Situação do complexo teníase humana e cisticercose no Brasil. Comunicações Cientificas da Faculdade de Medicina Veterinaria e Zootecnia da Universidade de São Paulo, São Paulo, v.8, p.227-229, 1984.

LAPAGE, G. Parasitologia veterinária. 6.ed. México, D.F.: Compañia Ed. Continental, 1981. p.259-285.

MINOZZO, J. C.; GUSSO, R. L. F.; CASTRO, E. A.; LAGO, O.; THOMAZ-SOCCOL, V. Experimental bovine infection with Taenia saginata eggs: recovery rates and cysticerci location. Brazilian Archives of Biology and Technology, Curitiba, v.45, n.4, p.451-455, 2002.

MIRANDA, Z. B. Inspeção de produtos de origem animal. Revista CFMV, Brasília, v.8, n.26, p.21-26, 2002.

PAIVA, D. P. Conhecendo a prevalência da cisticercose suína e bovina no Brasil: devemos rever nossos hábitos alimentares? CONGRESSO BRASILEIRO DE MEDICINA TROPICAL, 31., 2005, Florianópolis; ENCONTRO DE MEDICINA TROPICAL DO CONE SUL, 1., 2005, Florianópolis. Anais... Florianópolis: [s.n.], 2005.

PEREIRA, M. A. V.; SCHWANZ, V. S.; BARBOSA, C. G. Prevalência da cisticercose em carcaças de bovinos abatidos em matadouros-frigoríficos do Estado do Rio de Janeiro, submetidos ao controle do serviço de inspeção federal (SIF-RJ), no período de 1997 A 2003. Arquivos do Instituto Biológico, São Paulo, v.73, n.1, p.83-87, 2006.

PESSÔA, S. B.; MARTINS, A. V. Parasitologia médica. 11.ed. Rio de Janeiro: Guanabara Koogan, 1982.

REIS, D. O.; MUNDIM, M. J. S.; CABRAL, D. D.; CRUZ, J. M. C. Cisticercose bovina: 15 anos de ocorrência em animais abatidos em Uberlândia, 1979 a 1993. Higiene Alimentar, São Paulo, v.10, n.43, p.33-35, 1996.

RODRIGUES, L. V. C. Inspeção sanitária e critério de julgamento da cisticercose bovina calcificada: Infecção leve. Ciência Rural, Santa Maria, v.23, n.3, p.339-344, 1993.

SANTOS, I. F. Diagnóstico da cisticercose bovina em matadouros. III. Exame dos pilares diafragmáticos. Higiene Alimentar, São Paulo, v.7, n.25, 1993a.

Diagnóstico da cisticercose bovina em matadouros. II. Exame do diafragma. Arquivos Fluminenses de Medicina Veterinária, Niterói, v.2, n.3, p.72-8, 1987.

Um modelo de inspeção para a detecção da cisticercose muscular bovina em matadouros. 1993b. Tese (Livre Docência) - Universidade Federal Fluminense, Faculdade de Veterinária, Niterói.

SCHANTZ, P. M.; MOORE, A. C.; MUÑOZ, J. L.; HARTMAN, B. J.; SCHAEFER, J. A.; ARON, A. M.; PERSAUD, D.; SARTI, E.; WILSON, M.; FLISSER, A. Neurocysticercosis in an orthodox Jewish community in New York City. New England Journal of Medicine, Waltham, v.327, n.10, p.692-695, Sep. 1992.

SOUZA, R. M.; ANTUNES, C. F.; GUSATIMOSIN, C. B. A importância do serviço de inspeção federal na vigilância sanitária de alimentos - Cisticercose bovina. Higiene Alimentar, São Paulo, v.11, n.48, p.19-21, 1997.

UNGAR, M. L.; GERMANO, M. I. S.; BIGGI, G. S.; GERMANO, P. M. L. O valor dos registros de estabelecimentos de abate para a saúde pública. Comunicações Cientificas da Faculdade de Medicina Veterinaria e Zootecnia da Universidade de São Paulo, São Paulo, v.14, p.161-165, 1990. 